\title{
Hyperinsulinaemia accelerates accumulation of cholesterol ester in aorta of rats with transplanted pancreas
}

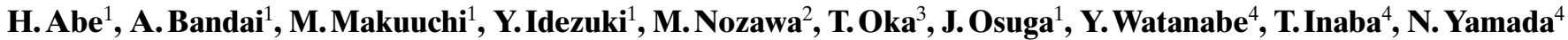 \\ ${ }^{1}$ Second Department of Surgery, University of Tokyo, Tokyo, Japan \\ ${ }^{2}$ Department of Surgery, Meikai University, Saitama, Japan \\ ${ }^{3}$ Department of Pathology, University of Tokyo, Tokyo, Japan \\ ${ }^{4}$ Third Department of Internal Medicine, University of Tokyo, Tokyo, Japan
}

\begin{abstract}
Summary Hyperinsulinaemia may play a role in the development of atherosclerosis; however, the direct effect of endogenous insulin on the atherosclerotic process is not well understood. To clarify this situation we performed pancreas transplantation with systemic venous drainage in Wistar Shionogi (WS) and Spontaneous Hypertensive (SHR) rats. Both rats received syngeneic pancreaticoduodenal transplants from donor rats. SHR rats were used to observe the additive effects of both hypertension and hyperinsulinaemia on the atherosclerotic process. Peak blood insulin levels after a glucose load were approximately two times higher in transplanted rats than in nontransplanted WS and SHR rats. By contrast, there was no difference in plasma glucose responses between transplanted and non-transplanted rats. Hyperinsulinaemia was not related to dyslipidaemia and hypertension in transplanted rats. Nine months after transplantation, the cholesterol ester contents of the
\end{abstract}

aortas of both WS and SHR transplanted rats were significantly higher than in the control rats (WS: $1.9 \pm 1.0$ vs $3.8 \pm 2.1 \mathrm{mg} / \mathrm{g}$ dry tissue, $p<0.01$; SHR: $1.7 \pm 1.3$ vs $3.7 \pm 1.4 \mathrm{mg} / \mathrm{g}$ dry tissue, $p<0.05)$. No differences were demonstrated in the thickness of the intima or in the histology of the aortas of transplanted and control rats. To study the mechanism for cholesterol ester accumulation in the arterial wall, we measured neutral cholesterol ester hydrolase activities in vascular medial smooth muscle cells. Insulin significantly suppressed neutral cholesterol ester hydrolase activities in medial smooth muscle cells. Our results indicate that endogenous hyperinsulinaemia contributes to the development of atherosclerosis by accelerating cholesterol ester accumulation in the arterial wall. [Diabetologia (1996) 39: [1276-1283]

Keywords Hyperinsulinaemia, pancreas, transplantation, atherosclerosis, cholesterol.
Atherosclerotic disorders such as coronary artery disease and cerebral vascular disease are a major cause of death in diabetic patients [1]. Various risk factors for atherosclerosis have been identified from epidemiological studies $[2,3]$. In particular, hyperinsulinaemia which is usually related to insulin resistance is considered to be an important risk factor for

Received: 27 March 1996 and in revised form: 8 July 1996

Corresponding author: Dr. N. Yamada, Third Department of Internal Medicine, University of Tokyo, 7-3-1 Hongo, Bunkyo-ku, Tokyo 113, Japan

Abbreviations: HDL, High density lipoprotein; WS, Wistar Shionogi; SHR, Spontaneous Hypertensive Rat; DMEM, Dulbeccos modified Eagle's medium; FCS, fetal calf serum. coronary artery disease [4-6]. The insulin-resistant state with hyperinsulinaemia often clusters with other risk factors including hypertension, glucose tolerance, obesity, hypertriglyceridaemia, and low plasma HDL-cholesterol levels, increasing the risk of cardiovascular disease, presumably due to promotion of atherosclerosis [4-6]. In addition, it has been suggested that insulin plays a role as a growth factor for vascular smooth muscle cells $[7,8]$ and has direct effects on the arterial wall during the process of atherosclerosis $[9,10]$. Several studies have demonstrated that exogenous insulin accelerates the progression of atherosclerosis [11-17]. However, exogenous insulin administration is not an ideal way to observe the direct effects of insulin on the atherosclerotic process, because excess insulin causes secretion of 
counter-regulatory hormones and sometimes results in pathological responses including hypoglycaemia [18]. To create an in vivo model of endogenous hyperinsulinaemia without the adverse effects of insulin resistance and unphysiological high insulin levels, we performed pancreas transplantations, in which rats received a syngeneic whole pancreas. Pancreas transplantation is considered to be an ideal treatment for insulin-dependent diabetes mellitus to prevent the development of diabetic vascular complications by correcting the insulin-deficient state; however, the possible progression of atherosclerosis after pancreas transplantation has also been suggested [19]. The present study was carried out to ascertain whether endogenous insulin itself accelerates the progression of atherosclerosis by minimizing the effects of insulin resistance on atherogenesis.

\section{Materials and methods}

Animals and pancreas transplantation. Male, 8-10-week-old Wistar Shionogi (WS) rats weighing 250-300 g, and male 1012-week-old Spontaneous Hypertensive (SHR) rats weighing 250-300 g were used for pancreas transplantation. SHR rats were used to observe the effect of hypertension on atherosclerosis. Recipient rats received syngeneic whole pancreas which was isolated from donor rats of similar age, sex and weight. All the rats were fed normal laboratory chow ad libitum.

Pancreatico-duodenal transplantation in rats was performed as described by Lee et al. [20]. In brief, the whole pancreas and duodenum were removed under ether anaesthesia and infused with heparinized $154 \mathrm{mmol} / \mathrm{l} \mathrm{NaCl}$. The graft was then transplanted heterotopically by anastomosis of the donor aorta to recipient aorta, donor portal vein to recipient inferior vena cava (by heterotopic transplantation), and duodenum to duodenum in an end-to-side fashion, therefore, recipient rats had two pancreas, both original and transplanted. Sham-operation was performed on control WS and SHR rats.

Measurement of glucose, insulin blood lipids, body weight and blood pressure. Blood samples were obtained from a tail vein under ether anaesthesia and the concentrations of plasma glucose and insulin were determined in the morning ad libitum 2 , 4,6 , and 8 months after transplantation. Plasma glucose levels were measured enzymatically and plasma insulin and glucagon levels were measured by a double antibody radioimmunoassay $[21,22]$. Furthermore, rats were fasted overnight and underwent an intravenous glucose tolerance test at 6 months in WS rats (six sham-operated and six transplanted rats), and at 9 months in SHR rats (four sham-operated and four transplanted rats). Under chloral hydrate anaesthesia $(300 \mathrm{mg} / \mathrm{kg}$, intraperitoneally), each rat was injected with glucose $(1.5 \mathrm{~g} / \mathrm{kg}$ body weight) into the dorsal penile vein. Blood samples were collected from the external jugular vein to evaluate plasma insulin and glucose responses after the glucose load. All of the samples were drawn using heparinized syringes before and 15 , $30,60,90,120$ and $180 \mathrm{~min}$ after the injection of glucose. The plasma was immediately separated, frozen and stored at $-20^{\circ} \mathrm{C}$ until the time of measurement.

Plasma cholesterol, HDL-cholesterol, and triglyceride concentrations were measured enzymatically 9 months after transplantation [23-25]. Body weight was also determined 9 months after transplantation and blood pressure was measured at a tail artery using an automatic machine 8 months after transplantation [26].

Histologic study. Nine months after transplantation, animals were killed under ether anaesthesia. The entire aorta from the aortic valve to the iliac bifurcation, except the site of the anastomosis, was removed and carefully cleaned of loose adventitial tissue. The portions of ascending aorta adjacent to the heart and descending aorta distal to the left subclavian artery were used for microscopic examinations. Samples for microscopic examination were punched out from two random sections of each ascending and descending aorta, fixed in $10 \%$ formaldehyde, and stained with haematoxylin and eosin. We measured the thickness of the intima and the media at three randomly selected sites per section of aorta in each SHR rat, and intima and media were defined as the area between the inner border of the endothelium and the inner border of the internal elastic membrane and the area between the inner border of the internal elastic membrane and the outer border of the external elastic membrane, respectively. We found no apparent macroscopic changes in the aortas of WS control and WS transplanted rats.

We measured the cholesteryl ester content of the whole aorta [27]. Each aorta was dried by lyophilization and stored at $-20^{\circ} \mathrm{C}$ until analysis. Total lipid extracts were prepared by cutting each aorta into small pieces and homogenizing three times in chloroform/methanol $(2: 1)$ in a tapered glass-glass homogenizer. Determinations of total and free cholesterol in the extracts were performed in triplicate by enzymatic assay [27].

Neutral cholesterol ester hydrolase. Medial smooth muscle cells were explanted from the intact rabbit aorta by the method of Fischer-Dzoga et al. [28]. The cells were passaged three times by trypsinization and seeded in Dulbecco's modified Eagle's medium (DMEM) containing 10\% fetal calf serum (FCS) in 6-cm dishes. Subconfluent cells were cultured with serum free medium for $24 \mathrm{~h}$ and then the medium was changed to DMEM containing $0.1 \%$ FCS in the presence of 10 or $100 \mathrm{nmol} / \mathrm{l}$ insulin. After incubation at $37^{\circ} \mathrm{C}$ for the indicated hours, cells were washed three times in cold phosphate buffered saline and scraped off dishes into sucrose buffer $(10 \mathrm{mmol} / \mathrm{l}$ Tris- $\mathrm{HCl}$ buffer, ph 7.5 containing $250 \mathrm{mmol} / \mathrm{l} \mathrm{su}-$ crose and $0.1 \mathrm{mmol} / \mathrm{l}$ EDTA). Cell homogenates prepared by sonication using a Sonifier 250 (Branson, Conn., USA) were assayed for neutral cholesterol ester hydrolase [29]. Enzyme activity is expressed as pmol of substrate hydrolyzed per hour per mg cell protein.

\section{Statistical analysis}

Data were analysed by the Wilcoxon-Mann-Whitney test and expressed as mean $\pm \mathrm{SD}$. $P<0.05$ was considered to be statistically significant.

\section{Results}

Body weight, blood pressure, and plasma lipids. At the beginning of experiments, body weights of WS control, WS transplanted, SHR control, and SHR transplanted rats were $271 \pm 10,271 \pm 16,281 \pm 14$, and $284 \pm 15 \mathrm{~g}$, respectively. Nine months after transplantation, the body weights in WS transplanted rats 
Table 1. Cholesterol contents in aorta and blood pressure (BP), body weight, and plasma lipid levels in control WS $(n=13)$, transplanted WS $(n=10)$, control SHR $(n=5)$, and transplanted SHR rats $(n=4)$

\begin{tabular}{|c|c|c|c|c|}
\hline & \multicolumn{2}{|l|}{ Wistar } & \multicolumn{2}{|l|}{ SHR } \\
\hline & control & transplant & control & transplant \\
\hline Body weight (g) & $407 \pm 16$ & $435 \pm 25^{\mathrm{c}}$ & $378 \pm 26$ & $418 \pm 9^{c}$ \\
\hline $\begin{array}{l}\text { Systolic BP } \\
(\mathrm{mmHg})\end{array}$ & $166 \pm 11$ & $163 \pm 12$ & $231 \pm 19$ & $209 \pm 23$ \\
\hline $\begin{array}{l}\text { Diastolic BP } \\
(\mathrm{mmHg})\end{array}$ & $119 \pm 12$ & $114 \pm 11$ & $161 \pm 7$ & $131 \pm 23$ \\
\hline $\begin{array}{l}\text { Triglyceride } \\
(\mathrm{mmol} / \mathrm{l})\end{array}$ & $1.16 \pm 0.37$ & $0.72 \pm 0.35^{\mathrm{b}}$ & $0.35 \pm 0.07$ & $0.37 \pm 0.12$ \\
\hline $\begin{array}{l}\text { Cholesterol } \\
(\mathrm{mmol} / \mathrm{l})\end{array}$ & $2.56 \pm 0.49$ & $2.20 \pm 0.49^{c}$ & $1.47 \pm 0.08$ & $1.29 \pm 0.36$ \\
\hline $\begin{array}{l}\text { HDL-cholesterol } \\
(\mathrm{mmol} / \mathrm{l})\end{array}$ & $1.53 \pm 0.57$ & $1.22 \pm 0.16$ & $0.72 \pm 0.13$ & $0.67 \pm 0.10$ \\
\hline CE/tissue (mg/g) & $1.9 \pm 1.0$ & $3.8 \pm 2.1^{\mathrm{b}}$ & $1.7 \pm 1.3$ & $3.7 \pm 1.4^{\mathrm{c}}$ \\
\hline $\begin{array}{l}\text { CE/tissue/TC } \\
{[\mathrm{mg} / \mathrm{g} /(\mathrm{mg} / \mathrm{ml})]}\end{array}$ & $2.0 \pm 1.1$ & $4.6 \pm 2.7^{\mathrm{a}}$ & $2.9 \pm 2.1$ & $7.7 \pm 3.2^{c}$ \\
\hline
\end{tabular}

Data are expressed as mean \pm SD. CE/tissue indicates cholesterol ester contents per gram dried tissue of aorta, respectively; values of $\mathrm{CE} /$ tissue/TC were obtained by dividing the cholesterol ester contents of the aorta $(\mathrm{CE} /$ tissue $)$ by the total plasma cholesterol concentrations $(\mathrm{mg} / \mathrm{ml})$.

${ }^{\mathrm{a}} p<0.005 ;{ }^{\mathrm{b}} p<0.01 ;{ }^{\mathrm{c}} p<0.05$ compared to control rats in either WS or SHR rats.

HDL cholesterol levels in WS control rats were significantly different from those in SHR control rats $(p<0.05)$

and SHR transplanted rats were significantly greater than those in WS control rats and SHR control rats, respectively (Table 1 ). There were no differences in blood pressure levels between WS control and WS transplanted rats and between SHR control and transplanted rats.

Nine months after transplantation, plasma total cholesterol and triglyceride levels were significantly greater in WS control rats than in WS transplanted rats, whereas there were no differences in plasma lipid levels between SHR transplanted and SHR control rats (Table 1).
Blood glucose and insulin. The plasma insulin concentrations of WS transplanted rats $(n=6)$ and SHR transplanted rats $(n=6)$ were significantly higher in each determination than those of WS control rats $(n=6)$ and SHR control rats $(n=6)$, respectively (Table 2). The plasma insulin concentrations of WS transplanted rats were $139 \%$ (transplant/control: $82 \pm 33 / 59 \pm 20 \mathrm{pmol} / \mathrm{l}$ ) to $179 \%$ (transplant/control: $102 \pm 43 / 57 \pm 19 \mathrm{pmol} / \mathrm{l})$ those of WS control rats, and those of SHR transplanted rats were $226 \%$ (transplant vs control: $181 \pm 63$ vs $80 \pm 25 \mathrm{pmol} / \mathrm{l}$ ) to $274 \%$ (transplant vs control: $208 \pm 76$ vs $76 \pm 37 \mathrm{pmol} / \mathrm{l})$ those of SHR control rats, whereas the plasma glucose concentrations of WS transplanted rats and SHR transplanted rats were not significantly different from those of WS control rats and SHR control rats, respectively, except those of SHR rats at 4 months after pancreas transplantation (Table 2). Similarly, during the intravenous glucose tolerance test, peak concentrations of plasma insulin in WS transplanted rats were $224 \%$ (transplant vs control: $121 \pm 63$ vs $54 \pm 14 \mathrm{pmol} / \mathrm{l}$ ) those of WS control rats, and those of SHR transplanted rats were $202 \%$ (transplant vs control: $200 \pm 74$ vs $99 \pm$ $35 \mathrm{pmol} / \mathrm{l}$ ) those of SHR control rats. Plasma glucose concentrations of WS transplanted rats and SHR transplanted rats were not significantly different from those of WS control rats and SHR control rats, respectively (Fig.1). The areas under the insulin concentration curves in WS transplanted rats were $163 \%$ (transplant vs control: 11541 vs 7101 pmol . $\mathrm{l}^{-1} \cdot \mathrm{min}^{-1}$ ) those of WS control rats, and those of SHR transplanted rats were $219 \%$ (transplant vs control: 15708 vs $7172 \mathrm{pmol} \cdot \mathrm{l}^{-1} \cdot \mathrm{min}^{-1}$ ) those of SHR control rats (Fig.1). Immunoreactive plasma glucagon levels in transplanted rats were apparently greater than those in WS control rats after 6 months (transplanted; $159.0 \pm 56.2$ vs control; $116.6 \pm$ $43.1 \mathrm{ng} / \mathrm{l}$ ) and 9 months (transplanted; $103.0 \pm 17.9 \mathrm{vs}$ control; $84.5 \pm 15.4 \mathrm{ng} / \mathrm{l}$ ) of pancreas transplantation, although the differences were not statistically significant. The areas under the glucagon concentration

Table 2. Blood glucose and insulin levels in rats fed normal laboratory chow ad libitum

\begin{tabular}{|c|c|c|c|c|c|}
\hline Months after transplantation & & 2 & 4 & 6 & 8 \\
\hline \multicolumn{6}{|l|}{ Wistar $(n=6)$} \\
\hline \multirow[t]{2}{*}{ Insulin (pmol/l) } & Control & $59 \pm 20$ & $57 \pm 19$ & $56 \pm 17$ & $58 \pm 23$ \\
\hline & Transplant & $82 \pm 33^{\mathrm{a}}$ & $102 \pm 43^{\mathrm{a}}$ & $96 \pm 35^{\mathrm{a}}$ & $88 \pm 17^{\mathrm{a}}$ \\
\hline & Transplant & $9.88 \pm 0.94$ & $9.99 \pm 1.61$ & $9.27 \pm 0.94$ & $9.71 \pm 0.33$ \\
\hline \multicolumn{6}{|l|}{$\operatorname{SHR}(n=6)$} \\
\hline Insulin $(\mathrm{pmol} / \mathrm{l})$ & Control & & $80 \pm 25$ & & $76 \pm 37$ \\
\hline & Transplant & & $8.83 \pm 1.39^{\mathrm{a}}$ & & $12.9 \pm 2.22$ \\
\hline
\end{tabular}

Values are mean \pm SD

${ }^{a} p<0.05$ compared to control. Insulin levels in WS control rats were significantly different from those in SHR control rats $(p<0.05)$ 


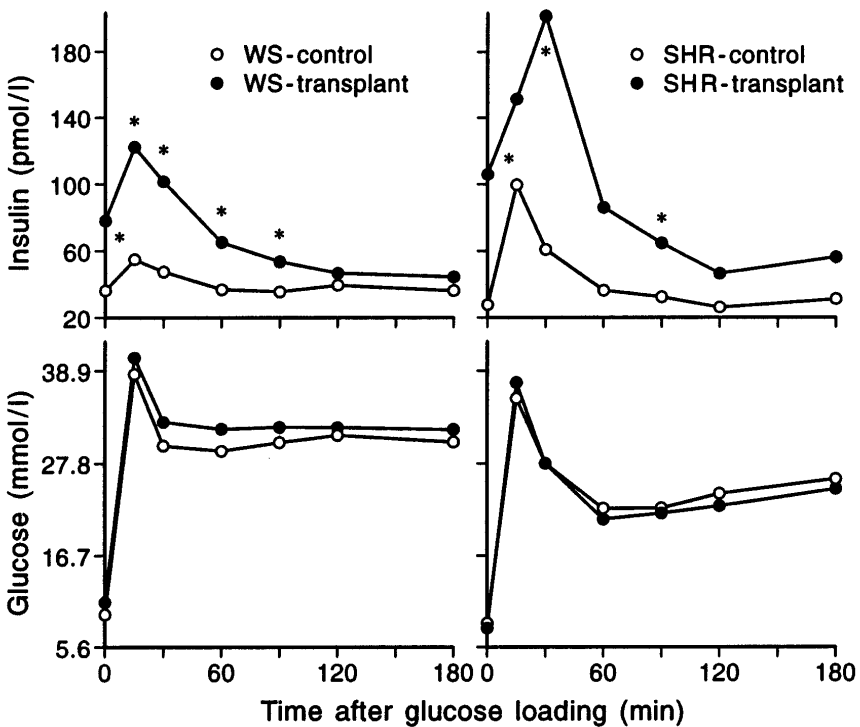

Fig.1. Blood glucose and insulin responses during intravenous glucose tolerance test (IVGTT). Rats were fasted overnight and underwent an IVGTT at 6 months in WS rats (six shamoperated and six transplanted rats), and at 9 months in SHR rats (four sham-operated and four transplanted rats). Rats were injected with $1.5 \mathrm{~g} / \mathrm{kg}$ body weight glucose into the dorsal penile vein. Blood samples were collected from the external jugular vein at the indicated times after a glucose load $* p<0.05$

curves in WS transplanted rats were not significantly different from those in WS control rats (transplant vs control: 18537 vs $\left.20365 \mathrm{ng} \cdot \mathrm{l}^{-1} \cdot \mathrm{min}^{-1}\right)$.

Cholesterol content of the aorta and microscopic examinations. Microscopic examination demonstrated no apparent increase in intimal thickness in both groups of WS rats (Fig. 2). By contrast, there was intimal thickening in aorta of SHR rats because of hypertension, however, no difference was observed in the intimal thickness between SHR control rats and SHR transplanted rats (Table 3). Immunohistochemical study with anti-rat monocyte-macrophage monoclonal antibody (Chemicon International Inc., Calif., USA) demonstrated no significant infiltration of macrophages in aorta of both WS and SHR transplanted rats as compared to control rats.

To evaluate atherogenic changes in aorta before histological changes developed, we measured aortic cholesterol content. We believe that measurement of cholesterol contents in aorta is more quantitative and sensitive for evaluating the extent of atherosclerosis than lipid staining, although lipid staining with oil red $\mathrm{O}$ was demonstrated in aorta of transplanted rats. The cholesterol ester content of the aorta was significantly greater in transplanted WS rats than in control WS rats. The cholesterol ester in transplanted $(n=10)$ and control WS rats $(n=13)$ was $3.8 \pm 2.1$ and $1.9 \pm 1.0 \mathrm{mg} / \mathrm{g}$ dry tissue, respectively $(p<0.01$, Table 1). Cholesterol ester in transplanted SHR rats was also high when compared to control rats $(p<0.05)$. When the aortic cholesterol ester content was corrected by the plasma cholesterol concentration $(\mathrm{mg} / \mathrm{ml})$ which is an important determinant of aortic cholesterol ester, the difference of the corrected values between transplanted rats and control rats was more evident than that of uncorrected values (Table 1). As shown in Figure 3, plasma insulin levels were positively correlated with cholesterol ester contents in the aorta, and those corrected values by plasma cholesterol levels were highly significantly correlated with plasma insulin levels.

Neutral cholesterol ester hydrolase activity. To clarify the mechanism for cholesterol ester accumulation in the arterial wall, we studied the effects of insulin on neutral cholesterol ester hydrolase activity in rabbit medial smooth muscle cells. As shown in Figure 4, both 10 and $100 \mathrm{nmol} / \mathrm{l}$ insulin significantly suppressed neutral cholesterol ester hydrolase activity in medial smooth muscle cells $(p<0.005)$ and the effect of insulin was dose-related. Four hours after incubation with $100 \mathrm{nmol} / \mathrm{l}$ insulin, neutral cholesterol ester hydrolase activity was suppressed by $70 \%$ as compared to that in the absence of insulin.

\section{Discussion}

Hyperinsulinaemia plays a role in the development of atherosclerosis through the pathophysiologic process related to insulin resistance, hyperlipidaemia, glucose intolerance, and hypertension [4-6]; however, the effects of insulin itself on atherogenesis are not clear. Animal models relevant to human hyperinsulinaemia and insulin resistance such as the $\mathrm{db} / \mathrm{db}$ mouse, ob/ob mouse [30], and OLETF rat [31] are not suitable for studying the effects of insulin on atherogenesis because hyperinsulinaemia is a result of insulin resistance in these animal models. Numerous animal experiments have demonstrated that insulin itself may contribute to the development of atherosclerosis by direct or indirect effects [11-17]. These animal experiments were performed using exogenous insulin injections with or without dietary modification in the presence or absence of diabetes. However, the atherogenic effects of exogenous insulin are controversial because non-physiological insulin injections administered to normal animals often cause pathologic responses such as hypoglycaemia [11, 12], and the secretion of counter-regulatory hormones including catecholamines [18] which may influence the atherosclerotic process.

Pancreas transplantation is a useful model with which to evaluate the effects of endogenous insulin on the artery by minimizing insulin resistance and the adverse effects of exogenous insulin injection. A well-regulated insulin secretion after glucose loading 


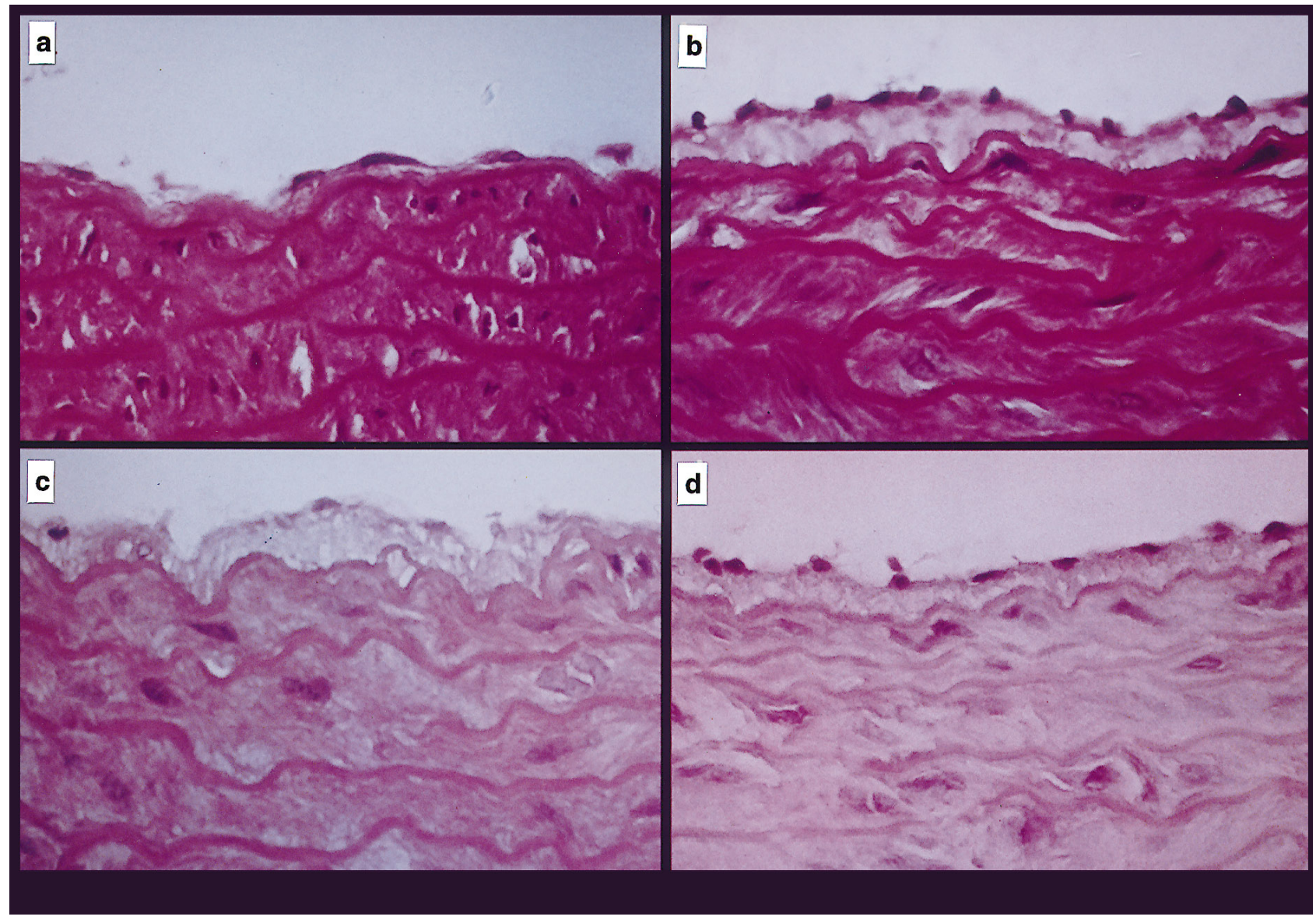

Fig. 2a-d. Light micrographs of the intima of aorta obtained from rats. (a) Ascending aorta of WS transplanted rat; (b) ascending aorta of SHR transplanted rat; (c) ascending aorta of SHR control rat; and (d) descending aorta of SHR transplanted rat. Magnification $\times 100$

Table 3. The thickness of the intima and media of the aorta obtained from SHR transplanted $(\mathrm{n}=5)$ and non-transplanted rats $(\mathrm{n}=5)$

\begin{tabular}{lllll}
\hline & & $\begin{array}{l}\text { Intima } \\
(\mu \mathrm{m})\end{array}$ & $\begin{array}{l}\text { Media } \\
(\mu \mathrm{m})\end{array}$ & $\begin{array}{l}\text { Intima/ } \\
\text { Media }\end{array}$ \\
\hline Ascending & Control & $41 \pm 5$ & $410 \pm 60$ & $0.10 \pm 0.01$ \\
aorta & Transplant & $40 \pm 5$ & $380 \pm 58$ & $0.11 \pm 0.03$ \\
Descending & Control & $35 \pm 12$ & $360 \pm 44$ & $0.10 \pm 0.03$ \\
aorta & Transplant & $30 \pm 8$ & $340 \pm 80$ & $0.09 \pm 0.02$ \\
\hline
\end{tabular}

Values are mean \pm SD

was observed in transplanted rats as well as in control rats. Although plasma insulin levels in transplanted rats were approximately twice as high as those in control rats, we found that plasma glucose levels in recipient rats were maintained at normal physiologic levels. Plasma glucose levels may be adjusted to normal by relatively high plasma glucagon levels in the transplanted rats. In addition, insulin resistance may be involved to some extent in the regulation of plasma glucose levels in transplanted rats, because hyperinsulinaemia possibly induces insulin resistance [32].

Plasma insulin concentrations were well correlated to cholesterol ester contents in the aorta, and the cholesterol ester content of the aorta was significantly greater in transplanted rats than in control rats. The corrected cholesterol ester content of aorta in SHR rats was higher than in WS rats (Table 1 ) because plasma insulin levels appear to be higher in SHR rats. Increased and regulated insulin secretion may affect cellular functions of the arterial wall and atherosclerotic processes. However, there was no microscopic evidence of early lesions of atherosclerosis in either WS or SHR rats, which are characterized by infiltration of monocyte-macrophages, migration of medial smooth muscle cells into the intima, proliferation of medial smooth muscle cells, and presence of foam cells [33]. Our results suggest that endogenous hyperinsulinaemia contributes to the development of atherosclerosis by accelerating cholesterol ester accumulation in the arterial wall, a characteristic pathological response in the atherosclerotic process. Thus, it is conceivable that endogenous hyperinsulinaemia determines the rate of cholesterol ester accumulation in the arterial wall rather than the cellular responses such as the migration and the proliferation of medial smooth muscle cells in the process of atherosclerosis. 


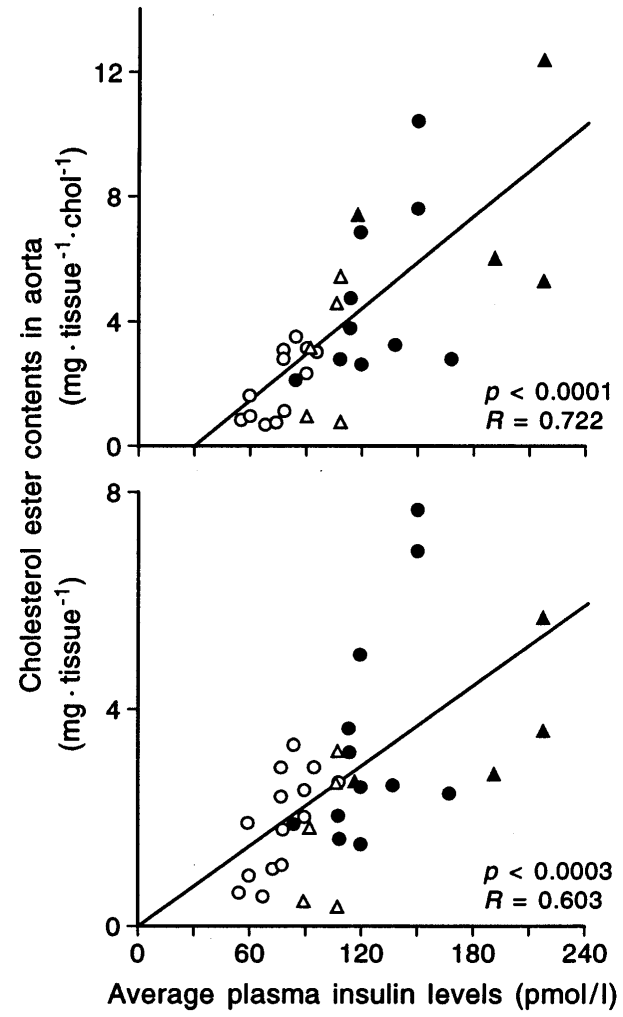

Fig. 3. Relationship between cholesterol ester contents in aortas and plasma insulin levels in rats. Accumulation of cholesterol ester in aorta of WS control (O), WS transplanted (•), SHR control $(\triangle)$, and SHR transplanted rats $(\boldsymbol{\Delta})$ is expressed as either cholesterol ester contents per gram dried tissue of aorta (lower) or the corrected values divided by average plasma cholesterol levels (upper). Plasma insulin and cholesterol levels were measured in the morning ad libitum 2, 4, 6 and 8 months after operation in WS rats and 4 and 8 months after operation in SHR rats. Average plasma insulin and cholesterol levels indicated the mean values of those 4 or 2 measurements in each rat

Insulin resistance is known to cause hyperinsulinaemia, glucose intolerance, hypertension, high plasma triglyceride, and low HDL-cholesterol concentrations in many population [4-6]. In our animal model, hyperinsulinaemia was not related to hyperlipidaemia, suggesting that insulin resistance is minimal. In contrast, we observed relatively low plasma lipid levels in transplanted WS rats as compared to control WS rats. High plasma insulin may reduce plasma lipid levels through effects on lipoprotein metabolism when insulin resistance is minimal, because insulin enhances triglyceride hydrolysis through increased lipoprotein lipase action [34], suppresses lipolysis with the decreased delivery of free fatty acids to the liver [35], enhances cholesterol removal through the increased low density lipoprotein receptor activity [36], and suppresses cholesterol absorption from the intestine through decreased acyl-coenzyme A: cholesterol acyltransferase activity [37]. In SHR rats, mild hyperinsulinaemia might not apparently

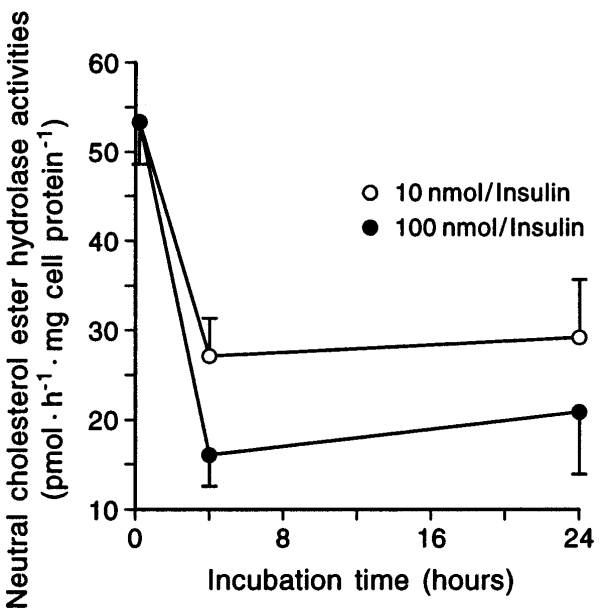

Fig. 4. The effects of insulin on the activity of neutral cholesterol ester hydrolase in rabbit aortic smooth muscle cells. The cells were cultured in $6 \mathrm{~cm}$ dishes with DMEM containing $10 \%$ FCS. In the experiment subconfluent cells were cultured with serum free medium for $24 \mathrm{~h}$ and then medium was changed to DMEM containing $0.1 \%$ FCS in the presence of 10 or $100 \mathrm{nmol} / \mathrm{l}$ insulin. After incubation at $37^{\circ} \mathrm{C}$ for the indicated hours, cells were scraped into sucrose buffer and sonicated. Cell homogenates were assayed for neutral cholesterol ester hydrolase. Enzyme activity is expressed as pmol of substrate hydrolysed per hour per mg cell protein. Each value represents the mean \pm SD of three experiments and each experiment was performed in triplicate. 10 and $100 \mathrm{nmol} / \mathrm{l}$ insulin significantly suppressed neutral cholesterol ester hydrolase activity after 4 and $24 \mathrm{~h}$ incubation $(p<0.005)$

influence the plasma lipid levels, since basal plasma lipid levels were much lower in SHR rats than in WS rats. Thus, this relative hyperlipidaemia in control WS rats may have reduced the difference in the accumulation of cholesterol ester in the aorta between control WS and transplanted WS rats, because plasma lipid levels are an important determinant of aortic cholesterol ester.

Body weights were slightly greater in transplanted rats than in non-transplanted rats. In view of the complex causal relationship between obesity and hyperinsulinaemia or insulin resistance this suggests hyperinsulinaemia may cause obesity by increasing lipid deposition when insulin resistance is minimal and obesity may reduce insulin sensitivity of peripheral tissues to some extent in transplanted rats.

Early atherosclerosis is characterized by the presence of foam cells in which lipoprotein-derived cholesterol esters accumulate [33]. At least six cellular mechanisms play a role in determining this cellular accumulation $[29,38]$. These include: (a) cellular synthesis of cholesterol, (b) the uptake of lipoproteincholesterol ester through specific receptors, (c) hydrolysis of lipoprotein-cholesterol ester by a lysosomal enzyme (acidic cholesterol ester hydrolase), (d) acyl-coenzyme A: cholesterol acyltransferase activity, (e) hydrolysis of cytosolic cholesterol ester by a non-lysosomal enzyme (neutral cholesterol ester 
hydrolase), and (f) mediation of the active excretion of cholesterol derived from the non-lysosomal hydrolysis of cytosolic cholesterol ester. Stout [11] has also suggested that increased synthesis of cellular cholesterol by insulin is involved in the accumulation of cholesterol esters in the aorta. Recent studies [39] have shown that hormone-sensitive lipase is identical to neutral cholesterol ester hydrolase. Since hormone-sensitive lipase activity is suppressed by insulin [40], neutral cholesterol ester hydrolase can also be suppressed by insulin, resulting in the accumulation of cholesterol ester during hyperinsulinaemia. Indeed, we and others demonstrated that $10 \mathrm{nmol} / \mathrm{l}$ insulin significantly suppressed neutral cholesterol ester hydrolase activity in vascular smooth muscle cells [41]. Taken together, hyperinsulinaemia may enhance cholesterol ester accumulation in the aorta both by stimulating cholesterol synthesis in the aorta and by suppressing hydrolysis of cholesterol ester.

In conclusion, endogenous hyperinsulinaemia influences the process of atherosclerosis by enhancing accumulation of cholesterol esters in the arterial wall without causing other features of the clinical metabolic syndrome in which high insulin levels are associated with hyperinsulinaemia, hypertension, and glucose intolerance. Therefore, hyperinsulinaemia should be minimized to prevent lipid accumulation in the arterial wall.

\section{References}

1. American Diabetes Association (1989) Role of cardiovascular risk factors in prevention and treatment of macrovascular disease in diabetes. Diabetes Care 12: 573-579

2. Zavaroni I, Dall'Aglio E, Bonora E, Alpi O, Passeri M, Reaven GM (1987) Evidence that multiple risk factors for coronary artery disease exist in individuals with abnormal glucose tolerance. Am J Med 83: 609-612

3. Kannel WB, Gordon T, Schwartz MJ (1971) Systolic versus diastolic blood pressure and risk of coronary artery disease: the Framingham study. Am J Cardiol 27: 335-346

4. Kaplan NM (1989) The deadly quartet. Arch Intern Med 149: $1514-1520$

5. Reaven GM (1988) Banting lecture 1988. Role of insulin resistance in human disease. Diabetes 37: 1595-1607

6. Zavaroni I, Bonora E, Pagliara M et al. (1989) Risk factors for coronary artery disease in healthy persons with hyperinsulinemia and normal glucose tolerance. N Engl J Med 320: 702-706

7. Stout RW, Bierman EL, Ross R (1975) Effect of insulin on the proliferation of cultured primate arterial smooth muscle cells. Circ Res 36: 319-327

8. Pfeible B, Ditschuneit H (1981) Effect of insulin on growth of cultured human arterial smooth muscle cells. Diabetologia 20: $155-158$

9. Stout RW (1969) Insulin stimulation of cholesterol synthesis by arterial tissue. Lancet II: 467-468

10. Chait A, Bierman EL, Albers JJ (1978) Regulatory role of insulin in the degradation of low density lipoprotein by cultured human skin fibroblasts. Biochim Biophys Acta 529: 292-299
11. Stout RW (1970) Development of vascular lesions in insulin-treated animals fed a normal diet. BMJ 19: 685-687

12. Sato Y, Shiraishi S, Oshida Y, Ishiguro T, Sakamoto N (1989) Experimental atherosclerosis-like lesions induced by hyperinsulinaemia in Wistar rats. Diabetes 38: 91-96

13. Stamler J, Pick R, Katz LN (1960) Effect of insulin in the induction and regression of atherosclerosis in the chick. Circ Res 8: 572-576

14. Duff GL, Brechin DJH, Finkelstein WE (1954) The effect of alloxan diabetes on experimental cholesterol atherosclerosis in the rabbit. IV The effect of insulin therapy on the inhibition of atherosclerosis in the alloxan diabetic rabbit. J Exp Med 100: 371-380

15. Mcgill HC, Holman RL (1949) The influence of alloxan diabetes on cholesterol atheromatosis in the rabbit. Proc Soc Exp Biol Med 72: 72-75

16. Cook DL, Mills MM, Green DM (1954) The mechanism of alloxan protection in experimental atherosclerosis. J Exp Med 99: 119-124

17. Wilson RB, Martin JM, Hartroft WS (1969) Failure of insulin therapy to prevent cardiovascular lesions in diabetic rats fed and atherogenic diet. Diabetes 18: 225-231

18. Boden G, Reichard GA, Hoeldetke RD, Rezvani I, Owen OE (1981) Severe insulin-induced hypoglycemia associated with deficiencies in the release of counterregulatory hormones. N Engl J Med 305: 1200-1205

19. Sutherland DER (1989) Effect of pancreatic transplantation on secondary complications of diabetes. In: Dubernard JM, Sutherland DER (eds) International handbook of pancreas transplantation. Kluwer Academic Publishers, Dordrecht pp 257-289

20. Lee S, Tung KS, Koopmans H, Chandler JG, Orloff MJ (1972) Pancreaticoduodenal transplantation in the rat. Transplantation 13: 421-425

21. Kadish AH, Litle RL, Sternberg JC (1968) A new and rapid method for determination of glucose by measurement of rate of oxygen consumption. Clin Chem 14: 116-131

22. Hales CN, Randle PJ (1963) Immunoassay of insulin with insulin antibody precipitate. Biochem J 88: 137-146

23. Allain CC, Poon LS, Chan CS, Richmond W, Fu PC (1974) Enzymatic determination of total serum cholesterol. Clin Chem 20: 470-475

24. Lopes-Virella HF, Stone P, Ellis S, Colwell JA (1977) Cholesterol determination in high-density lipoprotein separated by three different methods. Clin Chem 23: 882-884

25. Wahlfeld AW (1974) Triglyceride determination after enzymatic hydrolysis. In: Bergmeyer HU (ed) Methods of enzymatic analysis. New York Academic Press pp 1831-1835

26. Yamakoshi K, Shimazu H, Togawa T (1979) Indirect measurement of instantaneous arterial blood pressure in the rat. Am J Physiol 237: H632-H637

27. Yamada N, Inoue I, Kawamura M et al. (1992) Apolipoprotein $\mathrm{E}$ prevents the progression of atherosclerosis in WHHL rabbits. J Clin Invest 89: 706-711

28. Fisher-Dzoga K, Jones RM, Vesselinovitch D, Wissler RW (1973) Ultrastructural and immunohistochemical studies of primary cultures of aortic medial cells. Exp Mol Pathol 18: $162-176$

29. Inaba T, Shimano H, Gotoda T et al. (1993) Macrophage colony-stimulating factor regulates both activities of neutral and acidic cholesteryl ester hydrolases in human monocyte-derived macrophages. J Clin Invest 92: 479-485

30. Hunt CE, Lindseey JR, Walkley SU (1976) Animal models of diabetes and obesity, including the PBB/Ld mouse. Fed Proc 35: 1206-1217

31. Kawano K, Hirashima T, Mori S, Saitoh Y, Kurosumi M, Natori T (1992) Spontaneous long-term hyperglycemic rat 
with diabetic complications, Otsuka Long-Evans Tokushima Fatty (OLETF) Strain. Diabetes 41: 1422-1428

32. Rizza RA, Mandarino LJ, Genest J, Baker BA, Gerich JE (1985) Production of insulin resistance by hyperinsulinaemia in man. Diabetologia 28: 70-75

33. Ross R (1993) The pathogenesis of atherosclerosis: a perspective for the 1990s. Nature 362: 801-809

34. Brunzell JD, Porte D, Bierman EL (1979) Abnormal lipoprotein lipase mediated plasma triglyceride removal in untreated diabetes mellitus associated with hypertriglyceridemia. Metabolism 28: 901-907

35. Bierman EL, Dole VP, Roberts TN (1957) An abnormality of non-esterified fatty acid metabolism in diabetes mellitus. Diabetes 6: 475-479

36. Chait A, Bierman EL, Albers JJ (1978) Regulatory role of insulin in the degradation of low density lipoprotein by cultured human skin fibroblasts. Biochim Biophys Acta 529: 292-299
37. Jiao S, Matsuzawa Y, Matsubara K et al. (1988) Increased activity of intestinal acyl-CoA: cholesterol acyltransferase in rats with streptozotocin-induced diabetes and restoration by insulin supplementation. Diabetes 37: 342-346

38. Brown MS, Goldstein JL, Krieger M, Ho YK, Anderson RGW (1979) Reversible accumulation of cholesteryl esters in macrophages incubated with acetylated lipoproteins. J Cell Biol 82: 597-613

39. Small CA, Goodacre JA, Yeaman SJ (1989) Hormone-sensitive lipase is responsible for the neutral cholesterol ester hydrolase activity in macrophages. FEBS Lett 247: 205-208

40. Coppack SW, Jensen MD, Miles JM (1994) In vivo regulation of lipolysis in humans. J Lipid Res 35: 177-193

41. Fujiwara R, Shimada A, Tamai T, Nakai T, Miyabo S (1995) Effects of insulin, insulin-like growth factor-1, and phorbol esters on neutral cholesteryl esterase activity in cultured rat vascular smooth muscle cells. J Lab Clin Med 126: 240249 